\title{
Ensemble ecosystem modeling for predicting ecosystem response to predator reintroduction
}

Christopher M. Baker ${ }^{1 *}$, Ascelin Gordon ${ }^{2}$, and Michael Bode ${ }^{1}$

${ }^{1}$ School of BioSciences, The University of Melbourne, VIC, 3010, Australia

${ }^{2}$ School of Biological Sciences, University of Queensland, St Lucia Queensland, 4072, Australia

${ }^{2}$ School of Global, Urban and Social Studies, RMIT University, GPO Box 2476 Melbourne, 3001, VIC, Australia

*Current address: School of Biological Sciences, University of Queensland, St Lucia

Queensland, 4072, Australia, email c.baker7@uq.edu.au

Running title: Ensemble ecosystem modeling

Keywords: rewilding, Lotka-Volterra, dingo, wolf, trophic cascade

Word count: 5224

\begin{abstract}
Introducing a new, or previously extant, species to an ecosystem is a risky decision, and managers need quantitative methods that can predict the consequences for the recipient ecosystem. Proponents of keystone predator reintroductions commonly argue that the This article has been accepted for publication and undergone full peer review but has not been through the copyediting, typesetting, pagination and proofreading process, which may lead to differences between this version and the Version of Record. Please cite this article as doi: 10.1111/cobi.12798.
\end{abstract}

This article is protected by copyright. All rights reserved. 
presence of the predator will restore ecosystem function, but this has not always been the case, and mathematical modeling has important role to play in predicting how reintroductions will likely play out. We propose an ensemble modeling method that integrates species interaction networks and dynamic community simulations, and use it to describe the range of plausible consequences of keystone predator reintroduction. Previous methods for predicting ecosystem responses to such interventions have focused on predicting changes around a given equilibrium state and have conceptual problems extrapolating to new equilibria (e.g., with the new species present). Our method predicts changing abundances through time and works equally well when the system is far from equilibrium. We illustrate this method by modeling the range of potential outcomes for the reintroduction of wolves (Canis lupus) to Yellowstone National Park, and of dingoes (Canis dingo) to a National Park in Australia, and demonstrate how the method can provide important guidance on the potential risks of reintroduction.

Ensemble ecosystem modeling can also be applied to assess the ecosystem-wide implications of other types of interventions including assisted migration, biocontrol and invasive species eradication.

\section{Introduction}

The introduction of charismatic predators back into ecosystems they once inhabited has captured the imagination of scientists and the public (Marris 2014; Newsome \& Ripple 2015) - a prominent example being the reintroduction of wolves to Yellowstone National Park (Ripple \& Beschta 2012). Wolves are an example of a keystone species, playing an important role in ecosystem function and stability (Estes et al. 2011). Therefore, it is natural to think that keystone species reintroductions will improve ecosystem function (Ripple et al. 2014). However, reintroducing keystone species into ecosystems they previously inhabited can also 
lead to detrimental outcomes. For example, the wolf reintroduction in the northern Rockies of Idaho resulted in a rapid increase in wolf abundance and led to widespread human-wolf conflicts, the declaration of a disaster zone, and wolf culls (Stephens 2011; Gray et al. 2012). The dilemma of predator reintroduction is that, although the consequences are potentially positive, they are often uncertain. Despite this uncertainty, many conservationists continue to push for top predator reintroductions worldwide - from wolves and bears in North America, to wildcats in Europe and dingoes in Australia (Allen 2011; Jørgensen 2013; Chapron et al. 2014). Despite mixed results, predator reintroduction attempts are likely to continue, due to the charisma of the species and the backing of high-profile conservationists, scientists and managers (Marris 2014; Middleton 2014; Monbiot 2015; Buchanan 2015). It is therefore vital to develop methods capable of predicting the potential consequences for the recipient ecosystems, including a consideration of the risks and uncertainties in a systematic and transparent manner. Such predictions will support better decision-making, and help managers anticipate and respond to undesirable changes (Martin et al. 2012).

Predicting future ecosystem states requires a quantitative understanding of ecosystem dynamics. However, in many situations the available information - often provided in the form of food webs, or species interaction networks - is qualitative and conceptual (Hughes et al. 2013; Ripple et al. 2014; Newsome et al. 2015). Although relationships in the networks are established using quantitative data, this does not necessarily mean that the resulting models can make precise predictions about future states of an ecosystem. While it is possible to measure the strength of interactions between pairs of species, the sheer number of species and links in the relevant interaction networks make completion of this task unfeasible (Dambacher et al. 2003). In addition to the uncertain strength of the interactions, ecosystem dynamics are nonlinear and vary at a range of temporal scales. In almost all cases the system will be underdetermined, meaning that a wide range of future ecosystem dynamics will be 
consistent with the uncertain and incomplete information in the interaction network. This can result in greatly differing outcomes being plausible for each ecosystem component; in addition, the nonlinear interactions mean that initial population increases may turn into longterm declines (Strayer et al. 2006).

Due to the difficulty in predicting ecosystem responses to reintroduction, a range of mathematical methods have been developed that can handle qualitative and/or uncertain information. These originated with the qualitative models developed by Levins (Levins 1974, 1975). These models allow one to predict whether a species will increase or decrease following a change in the abundance of another species, using only information on whether species have a positive, negative or no interaction with each other. The underlying calculations are done analytically, but become overly cumbersome for larger communities involving approximately ten or more species. Hence, recent work has taken the approach of simulating vast numbers of quantitative community models that are consistent with the qualitative information, and using the range of outcomes to predict potential system responses (Raymond et al. 2011; Melbourne-Thomas et al. 2012). One of the key advantages of qualitative modeling is that there is no need to specify a functional form for species interactions. This is done by firstly linearising the system around its equilibrium point and then making predictions using the linearized system. While this gives qualitative modeling a useful generality, it is also a key weakness as it is not clear how far from equilibrium the linearized qualitative modeling predictions will be reliable. As species reintroductions may take a system far from its previous equilibrium state, new methods are required to predict how reintroductions may unfold. Some progress has been made using fuzzy cognitive mapping (Dexter et al. 2012; Hunter et al. 2015), as this method caters for species reintroductions, but it still only predicts equilibrium changes. 
In this paper we present our method of ensemble ecosystem modeling (EEM), and we demonstrate how it can systematically transform conceptual and uncertain descriptions of ecosystems via interaction networks into quantitative, risk analysis tools. We illustrate the method in the context of reintroducing keystone species, and show how it can be used to predict the range of plausible outcomes that may result. Our EEM approach builds on methods from Bayesian computation (Marjoram et al. 2003), qualitative modeling (Dambacher et al. 2003; Raymond et al. 2011) and ensemble methods in systems biology (Battogtokh et al. 2002). It provides a framework through which ambiguous interaction networks can be turned into an ensemble of predictions that, in aggregate, capture our understanding of the ecosystem dynamics, while faithfully representing our uncertainty. We use EEM to examine two high-profile qualitative interaction networks (Fig. 1) that were used to explain the consequences of reintroducing wolves to Yellowstone (Ripple et al. 2014) and dingoes to Australia's semi-arid rangelands (Newsome et al. 2015). We generate an ensemble of ecosystem models that are consistent with each network, and examine how their predictions about the consequences of reintroduction differ. We then show that large ensembles of these predictions can be partitioned into a finite and operationally-useful set of different post-reintroduction scenarios. Although the long-term consequences of reintroduction may take decades to emerge, we demonstrate that monitoring short-term changes in the abundance of key indicator species can dramatically reduce the uncertainty of long-term forecasts. These indicator species can therefore serve as early-warning signals for undesirable long-term outcomes.

\section{Methods}

EEM involves randomly generating hundreds of thousands of quantitative models, which are then filtered to retain only plausible realisations of a given qualitative interaction network. 
Throughout this paper we refer to the nodes in these networks as ecosystem elements, since they can represent individual species, groups of species, or abiotic attributes of the ecosystem like stream morphology or fire. For the networks in this paper, we use a system of generalised Lotka-Volterra equations to model species abundances through time. We choose this formulation as it is computationally straightforward, but still includes density dependent growth and interactions between all other species, and allows for a broad range of complex dynamics. We note that more complex models could be used within the EEM framework, either for individual species or the entire system. However, one must be careful when using more complex models, as they increase parameter space and do not necessarily lead to improved or different results (but see Wood 2001). We choose interaction terms whose signs reflect the network structure, but whose strengths are drawn randomly. This initial ensemble is then filtered by removing models that are inconsistent with plausible constrains on the system. For the examples presented here, we constrain ensemble members to be viable, meaning that all species can persist in the ecosystem (both with and without the predator; see Supplementary Materials). Each ensemble member is used to predict the ecosystem responses to predator reintroduction. This is done by calculating the trajectories for each network element, from its initial equilibrium before the reintroduction, to the new equilibrium with the predator present. Each model in the ensemble may make a different prediction, and any recommendations or conclusions can then be based on an analysis of the full range of simulated outcomes.

The generalised system of Lotka-Volterra equations used to model each ecosystem element through time is specified as follows (Murray 2002):

$$
\frac{d n_{i}}{d t}=r_{i} n_{i}+\sum_{j=1}^{N} \alpha_{i, j} n_{i} n_{j}
$$


Here $n_{i}$ is a measure of the abundance of ecosystem element $i$ (e.g., the number of individuals, their total biomass, their frequency in pit-fall traps), $r_{i}$ is growth rate of ecosystem element $i$, and $\alpha_{i, j}$ represents the strength of the per-capita interaction between ecosystem elements $i$ and $j$. To generate each ensemble member, the $r_{i}$ and $\alpha_{i, j}$ parameter values in Eq. (1) are drawn from probability distributions where the sign of the interaction is constrained to be consistent with the interaction network, and we assume that each node is self-limited $\left(\alpha_{i, i}<0\right)$. The results presented here are drawn from uniform distributions: $\left|\alpha_{i, j}\right| \sim U(0,1)$, but our results are robust to the distribution used (see Supplementary Materials section 1.5). We also constrain the predator-prey interactions weights, such that $\left|\alpha_{i, j}\right|<0.2 \times\left|\alpha_{j, i}\right|$, where $i$ is the predator and $j$ is the prey (see Supplementary Materials section 1.2; Bode et al. 2015). When information is available on species growth rates, the $r_{i}$ parameters can be drawn from uniform distributions with bounds based on the uncertainties in those growth rates, otherwise these parameters can be drawn from wide distributions, for example a uniform distribution bounded by the lowest and highest growth rates observed for any mammal species (Bode et al. 2015). We used growth rate estimates for some of the species, see Table S1 (Supplementary Materials). Structural uncertainty can also be included in this procedure. For example, in the dingo network (Fig. 1b), there are plausible arguments supporting either the presence or absence of a link between large herbivores and mesopredators. To include this uncertainty, the link can be included or excluded from each ensemble member at random (see Supplementary Materials). While similar arguments could be made about other links, we do this with a single interaction as a demonstration of how network uncertainty can be incorporated. 
Once a parameter set has been generated for an ensemble member, a viability check is made to test whether the set of parameters will result in a positive stable equilibrium. The first step in this is solving for the equilibrium state. Firstly, from the interaction matrix, $A$,

$$
A=\left(\begin{array}{cccc}
\alpha_{1,1} & \alpha_{1,2} & \cdots & \alpha_{1, n} \\
\alpha_{2,1} & \alpha_{2,2} & & \vdots \\
\vdots & & \ddots & \vdots \\
\alpha_{n, 1} & \cdots & \cdots & \alpha_{n, n}
\end{array}\right)
$$

and vector of growth rates, $\boldsymbol{r}$, where the $i^{\text {th }}$ element of $\boldsymbol{r}$ is $r_{i}$, then the equilibrium point, $n^{*}$, is

$$
n^{*}=-A^{-1} \boldsymbol{r}
$$

where $A^{-1}$ is the matrix inverse of $A$. If all elements of $n^{*}$ are positive, then a positive equilibrium exists. The next step is to determine whether the system is stable, which is done by assessing whether the real part of each eigenvalue of the associated Jacobian matrix (see Eq. (S9), Supplementary Materials) is negative. If both of these tests are passed, then the parameter set is viable. Other post hoc constraints can also be used to filter ensemble members. For example, if it were known that an increase in rainfall always coincides with an increase in the abundance of another species, the ensemble members that do not replicate these dynamics can be excluded (Raymond et al. 2011).

We choose to apply the viability constraint for both the pre- and post-reintroduction interaction networks. For the Yellowstone example, we know that none of the modelled species went extinct following the wolf reintroduction, implying the system was viable before and after wolf re-introduction. We make the same assumption for the dingo network, as each of the modelled ecosystem elements persist in ecosystems with and without dingoes. In general, the post-intervention viability assumption is not necessary, allowing ensemble members to be produced where species go extinct. 
Once the ensemble has been generated, the system perturbation (i.e., predator introduction) can be simulated. We simulate the introduction by adding the new species and its interactions into the system of equations specified in Eq. (1). We then solve Eq. (1) for the new steady state and directly solve for the trajectory between the old and new steady states. To simulate the dynamics, an initial condition must be specified, and we choose the equilibrium of the pre-reintroduction system as the initial condition for each ecosystem element. As species are generally introduced at low abundance, we set the initial condition of the new species to be $10 \%$ of the smallest equilibrium abundance of any species in the pre-reintroduction steady state.

The predictions of the final ensemble members can then be synthesised for use in a risk assessment for the intervention being modelled. This can involve determining the proportion of models in which a given ecosystem element increased or decreased between the old and new steady states; how long each element takes to reach the new steady state; and whether an initial increase (decrease) in a given component can be used to predict whether the final steady state has a higher or lower abundance. Finally, correlations between ecosystem elements across ensemble members reveal which ecosystem elements (if any) may be reliable indicators of the final system state.

\section{EEM algorithm overview}

Here we provide a short description of the EEM algorithm, which is provided in detailed in section S1.4 of the Supplementary Materials. To generate $M$ plausible models of a system:

1. Generate a random set of parameters $(A \& r)$.

2. Check for viability of the parameter set. 
3. If viable, add new node(s) to network and randomly generate parameters for the links to the new node(s).

4. Solve Eq. (1) to generate the transition to a new equilibrium.

5. Check for viability of the parameter set with the new nodes added, and store parameter set if it passes.

6. If fewer than $M$ models have been stored, return to step 1.

We have developed MATLAB code to simulate the EEM procedure, which is available at Figshare (see https://figshare.com/s/6d21abe2876511e5bb3006ec4bbcf141). This code simulates the reintroduction of a species to a network, and the dingo example is included in this code. See section S5 in Supplementary Materials for further details.

\section{Results}

To apply EEM to the Yellowstone network, we generated an ensemble of 100,000 viable models (which required us to generate and test 40 million candidate parameter sets). Each of the 11 species could potentially increase or decrease in response to the reintroduction: over 2,000 qualitatively different long-term responses. However, despite this potential diversity, plausible ecosystems exhibit only four qualitatively different ecosystem responses (Figure 2). The most frequent outcome of our model are the dynamics observed in Yellowstone National Park, which comprise an increase in the abundance of bears and woody plants, and an improvement in stream morphology (Ripple \& Beschta 2012). Further, the four different responses can be categorised on the basis of the relative change in two species' populations: bears and elks (Fig. 2).

Applying EEM to dingo reintroduction reveals a greater range of qualitatively different outcomes (Fig. 3). Out of the 10,000 viable models generated for the ensemble, dingo 
reintroduction caused a decrease in mesopredators and large herbivores in almost all models. However, the outcome for the other ecosystem elements appeared ambiguous (Fig. 3(a)). Nevertheless, categorising the ensemble predictions based on the dynamics of small vertebrates and invertebrates reveals a distinct pattern. When small vertebrates decrease, all other ecosystem components also decrease (Fig 3(a)), while an increase in invertebrate abundance is associated with an increase in the abundance of all other ecosystem components (Fig. 3(c)). This suggests that small vertebrates and invertebrates may be key indicator species to monitor, as their outcome could signal the dynamics of many other ecosystem components. We explored the uncertainty in whether mesopredators and large herbivores interact influenced these results. We included the link at random during the simulations and we found this uncertainty had no significant impact on our results (see Supplementary Materials).

Given that these systems are complex and uncertain, a monitoring regime that can costeffectively identify the changes in a wide range of ecosystem components is important, particularly because they take many years to reach a new equilibrium (Fig. 4). In some cases, a species that initially appears to increase, even over a 10 year period, can eventually decline below its pre-reintroduction abundance. In fact, the initial changes in the ecosystem gives almost no information about the equilibrium state. To show this, we separated the ensemble members into groups such that every member of the group had the same set of initial changes (e.g. mesopredator decrease, large herbivores decrease etc.). We then looked at the frequency of equilibrium outcomes in each group, and we found that the equilibrium outcomes were essentially unaffected by the initial changes in the system (see Supplementary Materials for further details). This may, in part, explain the differing results of studies that estimate the effect of dingoes on the ecosystem (Allen et al. 2013) and highlights the importance of 
targeted and model-guided monitoring in these situations, rather than just a greater monitoring effort (McDonald-Madden et al. 2010).

\section{Discussion}

In this paper we described ensemble ecosystem modeling (EEM) and used the approach to model the flow-on effects of predator reintroductions throughout the broader ecosystem. Our work demonstrates that even simple interaction networks can produce rich dynamics. This underscores the importance of using quantitative methods to predict ecosystem dynamics there are so many possible outcomes, we need to use systematic methods to synthesize results from ensembles of predictions. These results can support decision-making about reintroductions and assist targeted monitoring. Having quantitative predictions about the possible outcomes of a reintroduction is useful when deciding whether or not to proceed. Further, knowing the possible negative consequences of a reintroduction can help in prioritizing the formulation of recovery plans, by focusing on the species most at risk of a negative outcome. This approach can also help with prioritizing monitoring effort. For example, outcomes from the dingo case study indicate that monitoring small vertebrates and invertebrates is likely to deliver much more information than monitoring the other species in the interaction network.

Developing EEM models requires careful consideration of the interactions modelled in the ecosystem. Modeling every species individually would lead to unworkably cumbersome models, and networks should therefore be simplified. Certain species should be excluded and others grouped together, such that the network represents the key nodes and interactions present in the ecosystem. In some cases, there may be appropriate data to justify more complex functional forms (i.e., non-Lotka-Volterra models) for certain interactions. Although the interaction networks we have used are simplified versions of the full food web relevant to 
the predators in question, these networks are still able to provide insights into future outcomes, and these networks have been used in previous publications to explain and predict trophic cascades (Hughes et al. 2013; Ripple et al. 2014; Newsome et al. 2015).

In a sense, EEM is an extension of the established qualitative modeling (QM) approach (Dambacher et al. 2003; Raymond et al. 2011). There are some important differences between the two methods, notably that QM does not require the functional form of the relationship between any two species to be specified. There is also a difference in the outputs of the methods, QM predicts which species will decline or increase while EEM also predicts the size of the changes and the intermediate dynamics leading to these changes. However, the extra information that EEM provides comes at a cost. Firstly, EEM requires growth rate estimates, or distributions to sample growth rates from. Secondly, the magnitude of the perturbation must be specified. In this paper the magnitude of the perturbation is the initial abundance of the reintroduced species. While altering this does not change our equilibrium results, it would have an impact on the temporal dynamics. Finally, EEM is more computationally intensive than QM. One of the reasons for our choice of model, Eq. (1), is that it is computationally cheap to solve systems of Lotka-Volterra equations for the equilibrium state and its stability. However, we still have the additional cost of solving for the dynamics through time. Given the similarities between EEM and QM, it may be useful to run a QM analysis first, before using EEM if information about the magnitude of changes and dynamics was required.

Choosing the initial condition for the species being reintroduced is somewhat subjective and there is no obvious 'correct' way to do it. While in this paper we based set it to $10 \%$ of the smallest pre-reintroduction equilibrium abundance, other approaches could be used. For example the initial condition could be drawn at random from a distribution, such as between 
$5 \%$ and $50 \%$ of the smallest pre-reintroduction equilibrium abundance. The initial condition could also be chosen without the aid of known equilibria. Rather, it could be assumed that the new species will initially increase in abundance after being introduced. Hence, its initial density can be chosen randomly, with only ecosystems and initial conditions that exhibited an initial increase being retained in the ensemble. The distribution of initial densities could be a uniform distribution between 0 and $N_{m}$, where $N_{m}$ is chosen for a given ensemble member by numerically searching for the largest initial abundance that still results in an immediate increase.

The results of our EEM approach consist of ensembles of plausible future trajectories, from which we have reported the frequency of different classes of outcomes. Some authors advocate interpreting this type of result probabilistically in a Bayesian sense (Marjoram et al. 2003; Melbourne-Thomas et al. 2012). This interpretation assumes that model parameters the model priors - are drawn from distributions that accurately reflect our prior belief. We also ran our models with 16 different prior distributions (see Supplementary Materials), and found remarkably little variation in the results. Thus we believe our results can be interpreted probabilistically, but also somewhat skeptically. For example Raymond et al. (2011), use levels of 'support' to describe whether certain outcomes are likely or not (e.g. strong support; some support). We agree that it is reasonable to conclude that outcomes that occur more frequently than others in the simulations are more likely than others. However, generating precise probabilities of certain events are likely to be beyond the scope of this type of method.

Decisions around reintroductions are hard and EEM can provide assistance. However, EEM cannot make the decision; it can only make predictions about the range of future states of the system. Deciding whether to proceed or not is contingent upon value judgments. For example 
in the dingo case, would a predicted increase in small mammal and invertebrate abundance be sufficient to offset the predicted decrease in large herbivores? Different stakeholders may have differing opinions on the matter, and it is important to think carefully about the fundamental objectives of a project, and to use EEM in the context of those goals. Therefore, we do not advocate for or against dingo or wolf reintroduction here, as any potential location would have different species and ecosystem interactions and stakeholders with different goals and opinions.

The rewilding movement in modern conservation supports radical intervention into ecosystems, where locally extinct species or their analogues are reintroduced within their historical range (Marris 2014; Newsome et al. 2015). This same attitude advocates for the proactive movement of species threatened by climate change outside their historical range (McLachlan et al. 2007; Hoegh-Guldberg et al. 2008; Ricciardi \& Simberloff 2009), the eradication of invasive species from insular ecosystems (Veitch \& Clout 2002; Davis et al. 2011; Powell et al. 2013), and the introduction of biocontrol where eradication is not possible (Lowe et al. 2000; Messing \& Wright 2006; Shine 2010). All these situations are amenable to analysis with our EEM approach along with other types of ecosystem perturbations, such as the impacts of climate change through altering external drivers to the system, such as rainfall. These perturbations have uncertain consequences, and the strongest arguments ecosystem interventions often point out that ostensibly positive actions can unexpectedly cause severe negative consequences (Doak et al. 2008). These criticisms are plausible because our understanding of the relevant ecosystem dynamics is limited, and the level of uncertainty often appears overwhelming. While EEM does not entirely resolve all uncertainties, it provides a framework for transforming these uncertainties into a systematic analysis of the risks allowing decision-makers to view them in a structured and quantitative way. 


\section{Data Accessibility}

All data used in this paper were derived from publications, which are referenced where appropriate. We have provided MATLAB code for the EEM method, and the code is available from https://figshare.com/s/180dae6e88a427bb39b8.

\section{Acknowledgments}

This work was supported by the Australian Research Council (ARC) Centre of Excellence for Environmental Decisions and by ARC Discovery Project DP150102472. Christopher Baker is the recipient of a John Stocker Postdoctoral Fellowship from the Science and Industry Endowment Fund. Michael Bode was supported by DE130100572.

\section{Literature Cited}

Allen, B. L. 2011. A comment on the distribution of historical and contemporary livestock grazing across Australia: Implications for using dingoes for biodiversity conservation. Ecological Management \& Restoration 12:26-30.

Allen, B. L., P. J. S. Fleming, L. R. Allen, R. M. Engeman, G. Ballard, and L. K.-P. Leung. 2013. As clear as mud: A critical review of evidence for the ecological roles of Australian dingoes. Biological Conservation 159:158-174.

Battogtokh, D., D. K. Asch, M. E. Case, J. Arnold, and H.-B. Schüttler. 2002. An ensemble method for identifying regulatory circuits with special reference to the qa gene cluster of Neurospora crassa. Proceedings of the National Academy of Sciences 99:1690416909. 
Bode, M., C. M. Baker, and M. Plein. 2015. Eradicating down the food chain: optimal multispecies eradication schedules for a commonly encountered invaded island ecosystem. Journal of Applied Ecology:571-579.

Buchanan, R. T. 2015, March 24. David Attenborough backs calls for the reintroduction of wolves to Scottish highlands. Irish Independent. Dublin. Available from http://www.independent.ie/world-news/david-attenborough-backs-calls-for-thereintroduction-of-wolves-to-scottish-highlands-31091169.html (accessed June 19, 2015).

Chapron, G. et al. 2014. Recovery of large carnivores in Europe's modern human-dominated landscapes. Science 346:1517-1519.

Dambacher, J. M., H. W. Li, and P. A. Rossignol. 2003. Qualitative predictions in model ecosystems. Ecological Modelling 161:79-93.

Davis, M. A. et al. 2011. Don’t judge species on their origins. Nature 474:153-154.

Dexter, N., D. S. L. Ramsey, C. MacGregor, and D. Lindenmayer. 2012. Predicting Ecosystem Wide Impacts of Wallaby Management Using a Fuzzy Cognitive Map. Ecosystems 15:1363-1379.

Doak, D. F. et al. 2008. Understanding and predicting ecological dynamics: are major surprises inevitable. Ecology 89:952-961.

Estes, J. A. et al. 2011. Trophic Downgrading of Planet Earth. Science 333:301-306.

Gray, M., A. R. Krohn, M. K. Klip, R. A. Marsh, and L. A. McGinnis. 2012. The Big Bad Wolf or Symbol of the American Wilderness? Gray Wolf Reintroduction in Idaho.

Hoegh-Guldberg, O., L. Hughes, S. McIntyre, D. B. Lindenmayer, C. Parmesan, H. P. Possingham, and C. D. Thomas. 2008. Assisted Colonization and Rapid Climate Change. Science 321:345-346. 
Hughes, B. B., R. Eby, E. V. Dyke, M. T. Tinker, C. I. Marks, K. S. Johnson, and K. Wasson. 2013. Recovery of a top predator mediates negative eutrophic effects on seagrass. Proceedings of the National Academy of Sciences 110:15313-15318.

Hunter, D. O., T. Britz, M. Jones, and M. Letnic. 2015. Reintroduction of Tasmanian devils to mainland Australia can restore top-down control in ecosystems where dingoes have been extirpated. Biological Conservation 191:428-435.

Jørgensen, D. 2013. Reintroduction and De-extinction. BioScience 63:719-729.

Levins, R. 1974. Discussion Paper: The Qualitative Analysis of Partially Specified Systems. Annals of the New York Academy of Sciences 231:123-138.

Levins, R. 1975. Evolution in communities near equilibrium. Pages 16-60Ecology and evolution of communities. Harvard University Press, Cambridge.

Lowe, S., M. Browne, S. Boudjelas, and M. De Poorter. 2000. 100 of the World's Worst Invasive Alien Species A selection from the Global Invasive Species Database. Invasive Species Specialist Group, Aukland, New Zealand. Available from http://www.eea.europa.eu/data-and-maps/indicators/invasive-alien-species-ineurope/100-of-the-worlds-worst (accessed May 29, 2015).

Marjoram, P., J. Molitor, V. Plagnol, and S. Tavaré. 2003. Markov chain Monte Carlo without likelihoods. Proceedings of the National Academy of Sciences 100:1532415328.

Marris, E. 2014. Rethinking predators: Legend of the wolf. Nature 507:158-160.

Martin, T. G., S. Nally, A. A. Burbidge, S. Arnall, S. T. Garnett, M. W. Hayward, L. F. Lumsden, P. Menkhorst, E. McDonald-Madden, and H. P. Possingham. 2012. Acting fast helps avoid extinction. Conservation Letters 5:274-280. 
McDonald-Madden, E., P. W. J. Baxter, R. A. Fuller, T. G. Martin, E. T. Game, J.

Montambault, and H. P. Possingham. 2010. Monitoring does not always count.

Trends in Ecology \& Evolution 25:547-550.

McLachlan, J. S., J. J. Hellmann, and M. W. Schwartz. 2007. A Framework for Debate of Assisted Migration in an Era of Climate Change. Conservation Biology 21:297-302.

Melbourne-Thomas, J., S. Wotherspoon, B. Raymond, and A. Constable. 2012.

Comprehensive evaluation of model uncertainty in qualitative network analyses. Ecological Monographs 82:505-519.

Messing, R. H., and M. G. Wright. 2006. Biological control of invasive species: solution or pollution? Frontiers in Ecology and the Environment 4:132-140.

Middleton, A. 2014, March 9. Is the Wolf a Real American Hero? The New York Times. Available from http://www.nytimes.com/2014/03/10/opinion/is-the-wolf-a-realamerican-hero.html (accessed September 3, 2015).

Monbiot, G. 2015. 15 species that should be brought back to rewild Britain. Available from http://www.theguardian.com/environment/2015/jul/15/rewilding-britain-launcheswith-the-aim-of-restoring-uks-lost-wildlife-and-habitats (accessed September 3, 2015).

Murray, J. D. 2002. Mathematical biology I: An Introduction. Springer, New York; London.

Newsome, T. M. et al. 2015. Resolving the value of the dingo in ecological restoration. Restoration Ecology:201-208.

Newsome, T. M., and W. J. Ripple. 2015. Carnivore coexistence: Trophic cascades. Science 347:383-383.

Powell, K. I., J. M. Chase, and T. M. Knight. 2013. Invasive Plants Have Scale-Dependent Effects on Diversity by Altering Species-Area Relationships. Science 339:316-318. 
Raymond, B., J. McInnes, J. M. Dambacher, S. Way, and D. M. Bergstrom. 2011. Qualitative modelling of invasive species eradication on subantarctic Macquarie Island. Journal of Applied Ecology 48:181-191.

Ricciardi, A., and D. Simberloff. 2009. Assisted colonization is not a viable conservation strategy. Trends in Ecology \& Evolution 24:248-253.

Ripple, W. J. et al. 2014. Status and Ecological Effects of the World's Largest Carnivores. Science 343: 1241484 .

Ripple, W. J., and R. L. Beschta. 2012. Trophic cascades in Yellowstone: The first 15 years after wolf reintroduction. Biological Conservation 145:205-213.

Shine, B. R. 2010. The Ecological Impact of Invasive Cane Toads (Bufo Marinus) in Australia. The Quarterly Review of Biology 85:253-291.

Stephens, W. R. 2011. Gray Wolf Rising: Why the Clash over Wolf Management in the Northern Rockies Calls for Congressional Action to Define Recovery under the Endangered Species Act. William \& Mary Environmental Law and Policy Review 36:917.

Strayer, D. L., V. T. Eviner, J. M. Jeschke, and M. L. Pace. 2006. Understanding the longterm effects of species invasions. Trends in Ecology \& Evolution 21:645-651.

Veitch, C. R., and M. N. Clout. 2002. Turning the tide: the eradication of invasive species (proceedings of the international conference on eradication of island invasives). IUCN SCC Invasive Species Specialist Group, IUCN, Gland, Switzerland and Cambridge, UK.

Wood, S. N. 2001. Partially specified ecological models. Ecological Monographs 71:1-25.

\section{Figure legends}


Figure 1: Ecosystem interaction networks. (a) Yellowstone National Park, modified from Ripple et al. (2014) and (b) the dingo ecosystem, modified from Newsome et al. (2015). The reintroduced predator is shown as the top node in purple. The solid lines indicate interactions pre-reintroduction, and the dashed lines indicate the new interactions due to the reintroduction. Black arrows indicate a positive interaction and red arrows indicate a negative interaction, all of unknown strength. Predator-prey relationships are indicated by a positive and a negative arrow between two nodes (e.g. the wolf-elk link in (a)), while two black arrows indicates a mutualistic relationship (e.g. the invertebrates—grasses link in (b)). We note that there have been some minor modifications to the original dingo and Yellowstone networks: (1) The Yellowstone network only had one-way arrows, so arrows have been added to depict predator-prey relationships where appropriate; (2) we have omitted rain from the dingo network, as rain is not affected by any elements of the network; (3) we have removed an arrow from grass to soil, as the positive feedback from grasses to soil is included through the interactions with fire.
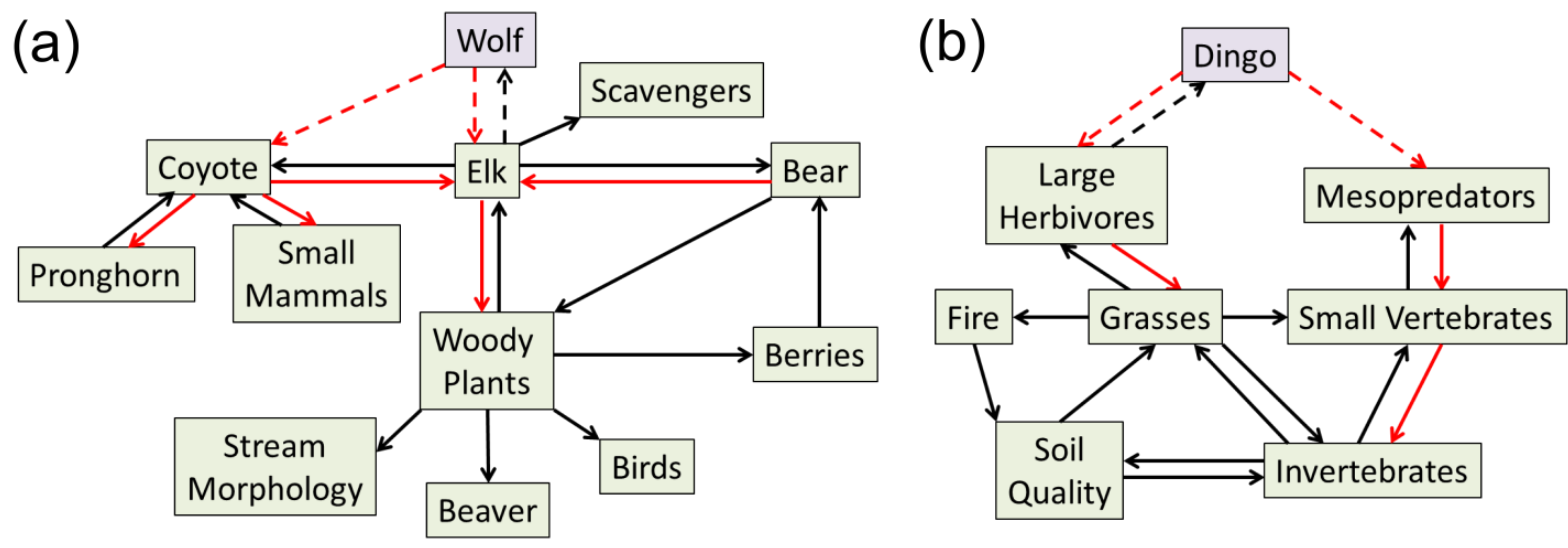

Figure 2: Equilibrium outcomes for the Yellowstone system. Light bars represent the proportion of models where an ecosystem element increased in size following wolf introduction, and dark bars represent the proportion of models where the element decreased. 
The results for all ensemble members are shown in (a) and subsets of the full ensemble are shown where elk increased (b), or decreased (c). EEM therefore predicts only four possible outcomes: all possible combinations of bears and elk increasing or decreasing.

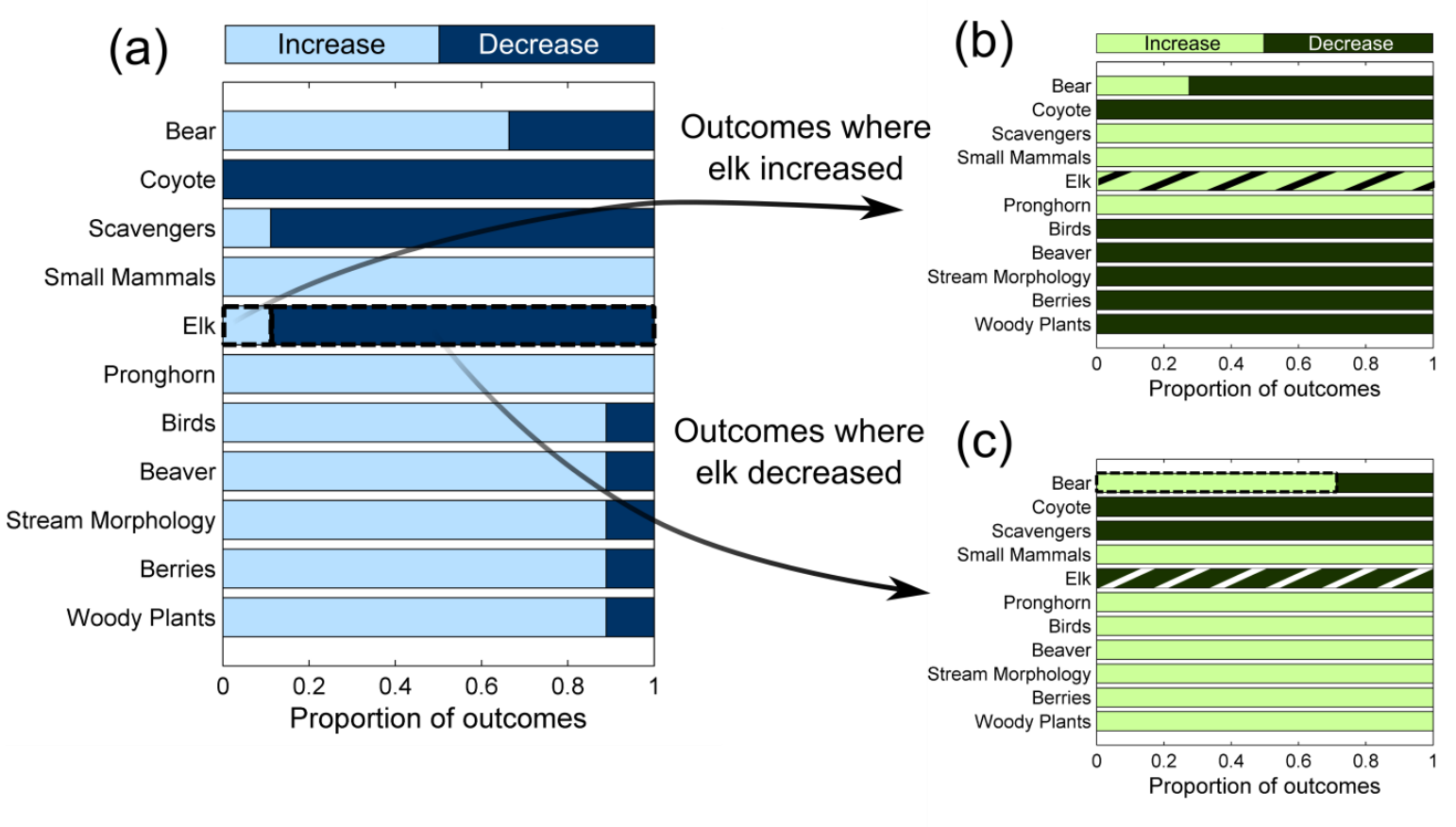

Figure 3: Equilibrium outcomes for the Dingo system. Light shading represents an increase in population size and while dark shading represents a decrease. Within these two categories, the shading is split to show larger changes (greater than 10\%; lightest/darkest shade) and small changes (less than 10\%). The results for all ensemble members are shown in (a), while (b) and (c) show the steady state outcomes when invertebrates increase, or when small vertebrates decrease respectively. Note that while the overall outcome (a) appears inconclusive - only mesopredators and large herbivores consistently decrease - there two circumstances which provide more concrete predictions. In scenarios where small vertebrates decline, most of the other ecosystem components decline; while when small vertebrates increase, all nodes except for large herbivores and mesopredators increase. 


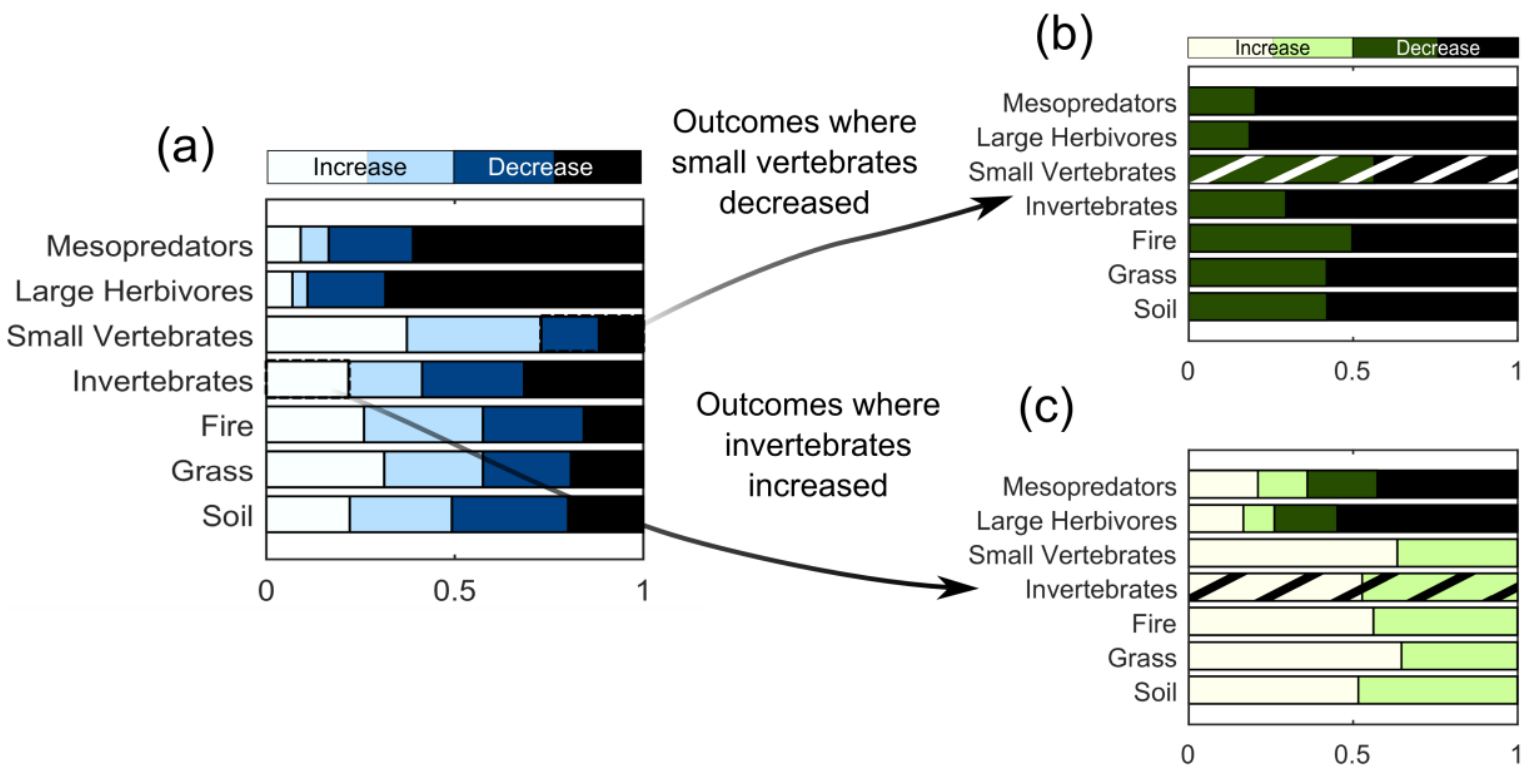

Figure 4: Waiting time before current observations align with long-term predictions for

dingo reintroduction. Ecosystem responses to reintroductions can be nonlinear (e.g., short-

term decreases can be followed by long-term increases). Even 5 years post-reintroduction, changes in the ecosystem align with the long-term outcomes in only $\sim 85 \%$ of ensemble members. Inset panels show the dynamics from different example ensemble members that align after various waiting times. For example, the middle inset depicts an ensemble member where large herbivores decline, relative to initial abundance, during the 12 years, even though their equilibrium abundance is higher than their pre-reintroduction levels (note where the purple line crosses the dashed line). 


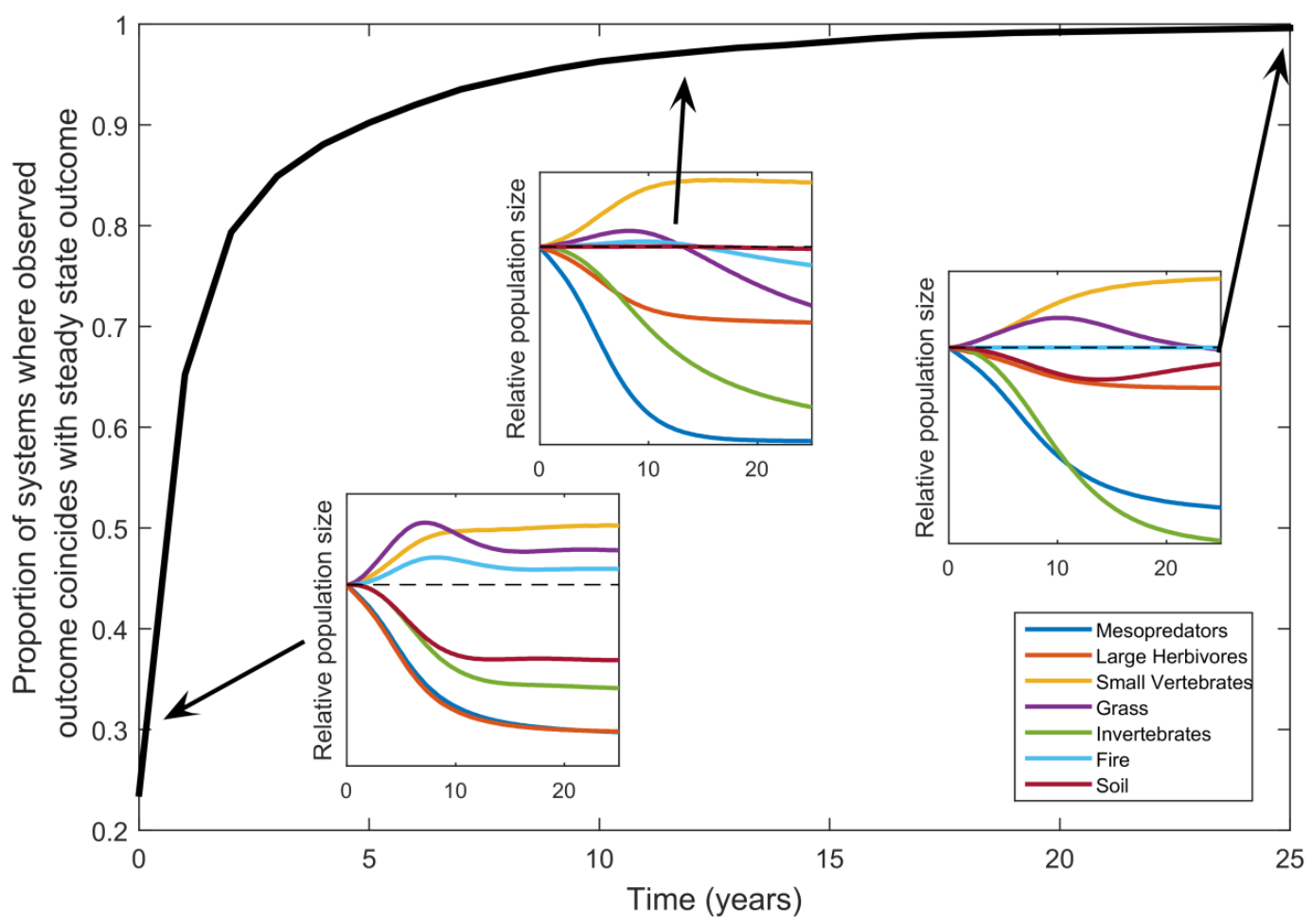

This article is protected by copyright. All rights reserved. 